Journal of Animal and Veterinary Advances 11 (8): 1255-1257, 2012

ISSN: $1680-5593$

(C) Medwell Journals, 2012

\title{
Anticoccidial Activity of Eupatorium adenophorum Extracts Against Chicken Coccidian Oocysts
}

\author{
${ }^{1,2}$ J.F. Yang, ${ }^{3}$ R.Q. Wang, ${ }^{1,2}$ R.Q. Lv, ${ }^{1}$ D.H. Zhou, ${ }^{2}$ G. Duan and ${ }^{1,2}$ F.C. Zou \\ ${ }^{1}$ State Key Laboratory of Veterinary Etiological Biology, \\ Chinese Academy of Agricultural Sciences, Lanzhou Veterinary Research Institute, \\ 730046 Lanzhou, Gansu Province, China \\ ${ }^{2}$ College of Animal Science and Technology, Yunnan Agricultural University, \\ 650201 Kunming, Yunnan Province, China \\ ${ }^{3}$ Department of Animal Husbandry and Veterinary, \\ Yunnan Agricultural Vocational Technical College, 650031 Kunming, Yunnan Province, China
}

\begin{abstract}
The potential anticoccidial activity of Eupatorium adenophorum extracts was evaluated against coccidian oocysts which are common parasites in poultry farms. Fresh unsporulated coccidian oocysts were exposed to $2,1.5,1$ or $0.5 \%$ of $E$. adenophorum extracts and the sporulation rate was measured after $72 \mathrm{~h}$. A total of 80 chickens were inoculated with those attenuated oocysts. After 1 week, the birds were challenged with $1 \times 10^{5}$ untreated fresh sporulated oocysts and the survival rate, Oocyst Per Gram feces (OPG), cecum lesion score, body weight gain rate and Anticoccidal Index (ACI) were measured and calculated on the 8th day after the challenge infection. The extracts of $E$. adenophorum were found to be significantly effective against coccidian oocyst activity $(\mathrm{p}<0.05)$. These results indicate that $E$. adenophorum may potentially be a new alternative for effective and environmentally-friendly control of coccidiosis. The results also have important implications for the development of attenuated coccidiosis vaccine.
\end{abstract}

Key words: Eupatorium adenophorum, chicken coccidian oocysts, anticoccidial activity, vaccine, survival rate, poultry farms

\section{INTRODUCTION}

Eupatorium adenophorum is a noxious weed that invades pastures and has spread to many countries over the past century (Sharma et al., 1998), reaching the Chinese province of Yunnan in the 1940s (Sun et al., 2004). The weed is also toxic to animals and may cause hoof or skin ulcerations, asthma, bronchitis, diarrhoea, dehairing, abortion and even death in livestock (Oelrichs et al., 1995; Sun et al., 2004). Various measures have been taken to eradicate and utilize the weeds such as making laminates, essence or dyes out of them ( $\mathrm{Lu}, 2007$; Lei et al., 2007). In previous studies, the extracts of $E$. adenophorum have been reported to be toxic to pests of crops and animal parasites or their eggs and intermediate hosts (Li et al., 2000, 2009; Zhang and Li, 2007; Zou et al., 2009).

However, no reports are available on the effects of such extracts against coccidian oocysts. Chicken coccidiosis is one of the most important poultry diseases with morbidity rates of $>80 \%$ in some poultry farms in
China. It accounts for annual loss of billions of US dollars for the poultry industry (Sun et al., 2009). Prevention and treatment of coccidiosis is mostly based on chemotherapy but the appearance of drug resistant strains has recently become a major threat (Chapman et al., 2010). Additionally, residues of the drug in the poultry raise serious health and safety concerns (Sharman et al., 2010). With public health commitment rising, alternative methods for controlling the parasite are much needed. One idea is to intervene at earlier points of control in the farm environment. For example, spraying disinfectants or insecticides in sheds to kill or inhibit the activity of oocysts and to end its life cycle at the sporulated stages can be an effective method. This is because such methods do not involve treating the animals themselves and can thus avoid problems related to drug resistance and residues.

The objective of the present study was to examine the anticoccidial activity of the extracts of the $E$. adenophorum plant and to develop a novel means for effective prevention and control of coccidiosis.

Corresponding Author: F.C. Zou, State Key Laboratory of Veterinary Etiological Biology, Chinese Academy of Agricultural Sciences, Lanzhou Veterinary Research Institute, 730046 Lanzhou, Gansu Province, China 


\section{MATERIALS AND METHODS}

Preparation of aqueous extracts of $E$. adenophorum whole plants of $E$. adenophorum were collected from the campus of Yunnan Agricultural University in Kunming, Yunnan province, China. The plants were washed with tap water and dried at $60^{\circ} \mathrm{C}$ before grinding them into powder and passing them through a 40 mesh $(0.35 \mathrm{~mm})$ sieve. A $500 \mathrm{~g}$ dried sample of the powder was mixed with about ten times the volume of $95 \%$ ethanol in a $65^{\circ} \mathrm{C}$ incubator for $48 \mathrm{~h}$, then passed through a filter paper with a pore size of $80-120 \mathrm{~mm}$. The filtrate was finally vacuum concentrated to obtain the final extracts. The extracts were diluted in double-distilled water to obtain final concentrations of $2,1.5,1$ and $0.5 \%$.

Chickens: Male Yunnan local chicks (1 day old) were purchased from the Poultry market at the Fengxiang Company (Kunming, China) and were fed in wire cages.

Coccidian oocysts: Coccidian oocysts were collected from infected chicks and identified as Eimeria tenella. The oocyts were then propagated in healthy 30 days old chickens.

Exposure of oocysts to the extracts: Unsporulated fresh oocysts were collected, purified and transferred to culture vessels. Culture vessels in the experimental group contained $5 \mathrm{~mL}$ of $2.5 \%$ potassium dichromate and $5 \mathrm{~mL}$ of either $2,1.5,1$ or $0.5 \%$ diluted extracts. The control group vessels consisted of $5 \mathrm{~mL} 2.5 \%$ potassium dichromate and $5 \mathrm{~mL}$ double-distilled water. Each experiment was performed in triplicates to ensure accuracy. All the group vessels were put in a $28^{\circ} \mathrm{C}$ incubator.

Observation of the sporulation rate: The efficacy of the $E$. adenophorum extracts in inhibiting oocyst development was recorded by observing the sporulation rate of the occidian oocysts in a counting chamber, after culturing the oocysts for $72 \mathrm{~h}$.

Observation of pathogenicity of attenuated coccidian oocysts: A total of 80 healthy chicks ( 30 days old) were divided into 4 groups and each group of 20 birds were inoculated with $500 \mathrm{mg}$ of sporulated oocysts which had been treated with $2,1.5,1$ or $0.5 \%$ E. adenophorum extracts. After 1 week, the four test groups and the positive control group, each consisting of 20 birds were challenged with $1 \times 10^{5}$ untreated sporulated oocysts while the another 20 birds that were not challenged were used as negative controls.
The efficacy of the attenuated oocysts in stimulating the immune system of the chicks to protect them against sporulated oocysts was evaluated on the basis of survival rate, Oocyst Per Gram feces (OPG) or oocysts value, cecum lesion score, body weight gain rate and the anticoccidal index (Johnson and Reid, 1970). All the indices were measured or calculated on the 8th day after challenge infection.

\section{RESULTS AND DISCUSSION}

Oocyst sporulation was significantly inhibited in groups treated with extracts of $E$. adenophorum compared with the control group $(\mathrm{p}<0.05)$. Using 2 or $1.5 \%$ of the extracts appeared more effective than using 1 or $0.5 \%$ of the extracts but the difference was not statistically significant ( $p>0.05$ ). Using $1 \%$ of the extracts appeared just as effective as $0.5 \%$ (Table 1 ).

Chicks in the experimental and the negative control groups survived after the challenge with unattenuated oocysts. The OPG of feces, measured on the 8th day after the challenge was clearly reduced with increase in concentration of the extracts. Groups that were treated with either 2 or $1.5 \%$ of the extracts were significantly inhibited effect and different from those in the $0.5 \%$ and positive control groups $(p<0.05)$. Similar results were obtained for cecum lesion score. The rate of body weight gain was higher with increase in concentration but not significantly different $(p>0.05)$. The ACI is a comprehensive indicator for anticoccidial effect and is calculated as (Table 1):

$$
\begin{aligned}
\mathrm{ACI}= & (\text { Relative weight gain rate }+ \text { Survival rate })- \\
& \text { (Lesion score } \times 10+\text { Oocyst value })
\end{aligned}
$$

Table 1: Anticoccidial activity of Eupatorium adenophorum against chicken

\begin{tabular}{|c|c|c|c|c|c|c|}
\hline \multirow[b]{2}{*}{ Groups } & \multicolumn{4}{|c|}{$\begin{array}{l}\text { Concentration of } E \text {. } \\
\text { adenophorum extract (\%) }\end{array}$} & \multicolumn{2}{|l|}{ Control } \\
\hline & 2.0 & 1.5 & 1.0 & 0.5 & Positive & Negative \\
\hline Sporulating rates $(\%)$ & 36.4 & 47.8 & 55.6 & 56.0 & 94.4 & 94.4 \\
\hline Survival rate (\%) & 100.0 & 100.0 & 100.0 & 100.0 & 62.5 & 100.0 \\
\hline OPG $(\times 100)$ & 54.0 & 72.0 & 108.0 & 121.0 & 198.0 & 0.0 \\
\hline Oocyst value & 10.0 & 20.0 & 20.0 & 20.0 & 40.0 & 0.0 \\
\hline Cecum lesion score (mean) & +1.4 & +1.4 & +2.3 & +2.8 & +3.5 & 0.0 \\
\hline Body weight gain rate (\%) & 98.3 & 96.9 & 82.8 & 79.7 & 73.0 & 100.0 \\
\hline Anticoccidal index & 174.3 & 162.9 & 139.8 & 131.7 & 60.5 & 200.0 \\
\hline
\end{tabular}
coccidian oocysts

Oocyst value base on the oocysts rate $=$ (Number of oocysts for test group/Number of oocysts for positive control group) $\times 100$. If the oocy sts rate range in $0-1 \%$ and the oocysts value requal to 0 if the oocysts rate range in $1-25 \%$ and the oocysts value requal to 5 if the oocysts rate range in $26-50 \%$ and the oocysts value requal to 10 if the oocysts rate range in $51-75 \%$ and the oocysts value requal to 20 if the oocysts rate range in $76-100 \%$ and the oocysts value requal to $40 . \mathrm{ACI}=$ (Relative weight gain rate+Survival rate)(Lesion score $\times 10+$ Oocyst value) 
The results showed that the test groups (groups treated with $E$. adenophorum extracts) and the negative group had significantly higher ACI than the positive group $(\mathrm{p}<0.05)$. This demonstrates that the $E$. adenophorum extracts exhibited strong anticoccidial activity by inhibiting the efficacy of coccidian oocyst development.

Current methods for prevention and control of coccidiosis mainly relies on the use of chemical drugs on farm animals and thus raises serious concerns about drug residues and food safety. Therefore, finding an alternative method of directly killing coccidians or inhibiting their oocyst activity in the external environment by using an efficient, biodegradable substance which is important. The use of plant-based insecticides in farm houses for prevention and control of coccidiosis is a promising alternative (Tafara et al., 2005). The results show that the extracts of $E$. adenophorum may be a novel plant-based, environmentally friendly pesticide or anticocccidial agent that lacks the health risks posed by chemical-based anticoccidial agents currently in use.

Previous studies have shown that the main active ingredients of $E$. adenophorum include triterpenes, flavonoids and phenylpropanoid phenolic compounds and these compounds have insecticidal activity (Yan et al., 2006).

\section{CONCLUSION}

The results are consistent with these observations and also provide a foundation for the utilization of $E$. adenophorum in the development of attenuated coccidiosis vaccine.

\section{ACKNOWLEDGEMENTS}

This study was financially supported in part by the Open Funds of the State Key Laboratory of Veterinary Etiological Biology, Lanzhou Veterinary Research Institute, Chinese Academy of Agricultural Sciences (SKLVEB2010KFKT009, SKLVEB2011KFKT010), the Yunnan Provincial Program for Introducing High-level Scientists (2009C1125) and the Program for Innovative Research Team of Yunnan Province (2011-14). Yang, Wang and Lv contributed equally to this research.

\section{REFERENCES}

Chapman, H.D., T.K. Jeffers andR.B. Williams, 2010. Forty years of monensin for the control of coccidiosis in poultry. Poult. Sci., 89: 1788-1801.
Johnson, J. and W.H. Reid, 1970. Anticoccidial drug: Lesion scoring techniques in battery nd floor-pen experiment. Exp. Parasitol., 29: 30-36.

Li, B., P.B. Zhao, J. Chen, S.R. Li and F.C. Zou, 2009. Effect of the soaking solution of Eupatoeium japonicum on river snail-killing. J. Anhui Agric. Sci., 37: 4069-4070.

Li, Y.S., H.Y. Zou, Z. Nai, W.Y. Li, X.Y. Na, S.Z. Tang and Y.Z. Yang, 2000. Insecticidal activity of different fractions of essential oils extracted from Eupatorium adenophorum against four species of stored grain insects. J. Southwest Agric. Univ., 22: 331-333.

Lu, C.C., 2007. Research status of Eupatorium adenophorum Spreng (an important alien species). Weed Sci., 2: 10-13.

Oelrichs, P.B., C.A. Calanasan, J.K. MacLeod, A.A. Seawright and J.C. Ng, 1995. Isolation of a compound from Eupatorium adenophorum (Spreng.) [Ageratina adenophora (Spreng.)] causing hepatotoxicity in mice. Nat. Toxins, 3: 350-354.

Sharma, O.P., R.K. Dawra, N.P. Kurade and P.D. Sharma, 1998. A review of the toxicosis and biological properties of the genus Eupatorium. Nat. Toxins., 6: $1-14$.

Sharman, P.A., N.C. Smith, M.G. Wallach and M. Katrib, 2010. Chasing the golden egg: Vaccination against poultry coccidiosis. Parasite Immunol., 32: 590-598.

Sun, X.M., W. Pang, T. Jia, W.C. Yan and G. He et al., 2009. Prevalence of Eimeria species in broilers with subclinical signs from fifty farms. Avian. Dis., 53: 301-305.

Sun, X.Y., Z.H. Lu and W.G. Sang, 2004. Review on studies of Eupatorium adenophorum: An important invasive species in China. Scand. J. Forest Res., 15: 319-322.

Tafara, M., F.M. John and F.K. Emily, 2005. The efficacy of banana plant (Musa paradisiacal) as a Coccidiostat in rabbits. Int. J. Appl. Res. Vet. M., 3: 326-331.

Yan, Q.S., J. Yang, H.M. Li, A.C. Cao, Q.H. Chen, Y.Q. Wen and L. He, 2006. Advances in the studies on the chemical components and bioactivity of Eupatorium adenophorum Spreng as an intruding species. J. Beijing Nor. Univ., 42: 70-71.

Zhang, Q.F. and S.R. Li, 2007. Effect of water extract of Eupatorium adenophorum stem on Ascaris suum. Chin. J. Trad. Vet. Sci., 1: 3-4.

Zou, F.C., G. Duan, Y.J. Xie, Y. Zhou, G.D. Dong, R.Q. Lin and X.Q. Zhu, 2009. Molluscicidal activity of the plant Eupatorium adenophorum against Oncomelania hupensis, the intermediate host snail of Schistosoma japonicum. Ann. Trop. Med. Parasit., 103: 549-553. 